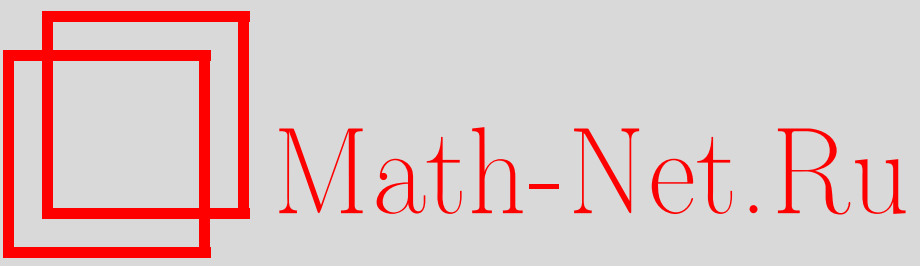

А. А. Герко, О структуре множества полудуализирующих комплексов, УМH, 2004, том 59, выпуск 5, 145-146

DOI: https://doi.org/10.4213/rm776

Использование Общероссийского математического портала Math-Net.Ru подразумевает, что вы прочитали и согласны с пользовательским соглашением

http://www.mathnet.ru/rus/agreement

Параметры загрузки:

IP : 52.205 .19 .152

26 апреля 2023 г., 14:29:20 


\title{
О СТРУКТУРЕ МНОЖЕСТВА ПОЛУДУАЛИЗИРУЮЩИХ КОМПЛЕКСОВ
}

\author{
А. А. ГЕРКо
}

Мы работаем в производной категории $\mathscr{D}_{b}^{f}(R)$ комплексов с конечным числом (конечнопорожденных) ненулевых гомологий над коммутативньм нётеровым локальным кольцом $R$. Комплекс $K \in \mathscr{D}_{b}^{f}(R)$ назьвается полудуализирующим (см. [1], [2]) если $\mathbf{R H o m}_{R}(K, K) \simeq R$. Тривиальными примерами таких комплексов являются свободный модуль ранга 1 и дуализирующий комплекс. С каждым полудуализирующим комплексом $K$ связан класс комплексов из $\mathscr{D}_{b}^{f}(R)$, имеющих конечную $G$-размерность относительно этого комплекса: мы говорим, что комплекс $X$ имеет конечную $G_{K}$-размерность, если $\operatorname{RHom}_{R}(X, K) \in \mathscr{D}_{b}^{f}(R)$ и морфизм $X \rightarrow \mathbf{R H o m}_{R}\left(\mathbf{R H o m}_{R}(X, K), K\right)$ является квазиизоморфизмом. В этом случае мы полагаем $G_{K} \operatorname{dim} X=-\inf \left(\mathbf{R} \operatorname{Hom}_{R}(X, K)\right)+\inf (K)$. Классическая $G$-размерность Ауслендера и Бриджера [3] соответствует случаю $K=R$. Необходимость в изучении структуры множества полудуализирующих комплексов возникает при исследовании поведения классической $G$-размерности при замене колец, в частности, непосредственно связано с вопросом о транзитивности $G$-размерности (см. [4]). Для всех известных автору примеров колец, над которыми существуют нетривиальные полудуализирующие комплексы (см. [5]), множество таких комплексов имеет естественную структуру частично упорядоченного множества, идентичную структуре множества всех подмножеств конечного множества. В настоящей работе анонсируются некоторые результаты, дающие основания полагать, что подобная структура может иметь место и в общем случае. Также мы получаем некоторые количественные и качественные результаты для артиновых колец, обладающих, в некотором смысле, максималњно возможным количеством полудуализирующих модулей.

Теорема 1. Если для полудуализирующих комплексов $X_{1}$ и $X_{2}$ над кольчом $R$ известно, что $G_{X_{2}} \operatorname{dim} X_{1}<\infty$, а комплекс $M$ имеет конечную $G$-размерность по отношению $X_{1}$ и $X_{2}$, то морфизм $\varphi: \operatorname{RHom}_{R}\left(M, X_{1}\right) \otimes{ }^{L} \mathrm{RHom}{ }_{R}\left(X_{1}, X_{2}\right) \rightarrow \operatorname{RHom} \operatorname{Hom}_{R}\left(M, X_{2}\right)$ является изоморфизмом.

ОпредЕлЕниЕ 2 . Неизморфные полудуализирующие комплексы $X_{0}, X_{1}, \ldots, X_{n}$ образуют чепочку длины $n$, если $G_{X_{i}} \operatorname{dim} X_{i-1}<\infty$ для всех $i=1, \ldots, n$.

СлЕДСтвиЕ 3. Если полудуализирующие комплексы $X_{0}, X_{1}, \ldots, X_{n}$ образуют чепочку, то имеет место изоморфизм

$$
X_{n} \simeq X_{0} \otimes^{L} \operatorname{RHom}_{R}\left(X_{0}, X_{1}\right) \otimes^{L} \mathrm{RHom} R\left(X_{1}, X_{2}\right) \otimes^{L} \cdots \otimes^{L} \mathrm{RHom} R\left(X_{n-1}, X_{n}\right) .
$$

В качестве следствия получаем, что не существует замкнутых цепочек полудуализирующих модулей, т.е. таких, где $X_{n} \simeq X_{0}$ (Для случая цепочек длины 1 с $X_{0} \simeq R$ это было показано в [1], а для произвольных цепочек длины 1 в работе [6]).

СледСТВиЕ 4. Если полудуализирующие комплексы $X_{0}, X_{1}, \ldots, X_{n}$ образуют чепочку и $X_{n} \simeq X_{0}$, то имеет место изоморфизм $X_{i} \simeq X_{j}$ для любых $i, j$.

Для примера из [5] цепочкам полудуализирующих модулей отвечают цепочки вложенных подмножеств, а следствию 1 - разложение максималшного подмножества в цепочке на объединение минимального и дополнений $i$-го подмножества до $(i+1)$-го.

Далее кольцо $R$ - артиново.

ГипотезА 5. Если полудуализирующие модули $K_{0}, K_{1}, \ldots, K_{n}$ образуют цепочку, то $\mathfrak{m}^{n} \neq 0$. Если, при тех же условиях, $\mathfrak{m}^{n+1}=0$, то ряд Пуанкаре $R$ имеет вид $\frac{1}{\prod_{i=1}^{n}\left(1-d_{i} t\right)}$ для некоторых положительньх чельх $d_{i}$.

Работа выполнена при частичной поддержке грантов РФФИ 02-01-00468 и НШ1910.2003.1 "Поддержка ведущих научных школ". 
Tеорема 6. Гипотеза 1 справедлива для $n \leqslant 3$.

ОПРЕДЕЛЕнИЕ 7 . Модули $K_{1}, \ldots, K_{n}$ назьваются сильно Tor-независимыми, если для любого $I \subset\{1, \ldots, n\}$ выполнено $\operatorname{amp}\left(\otimes_{i \in I}^{L} K_{i}\right)=0$.

ОПредЕЛЕниЕ 8 . Артиново колцо назьвается $S D(n)$-полным, если выполнены следующие условия:

(1) $\mathfrak{m}^{n+1}=0$;

(2) имеются сильно Tor-независимые несвободные полудуализирующие модули $K_{1}, \ldots, K_{n}$ такие, что для любого $I \subset\{1, \ldots, n\}$ модуль $\bigotimes_{i \in I}^{L} K_{i}$ - полудуализирующий.

ЗАмЕчАНиЕ 9 . Если множество полудуализирующих модулей удовлетворяет условию (2) из определения 8 , то полудуализирующие модули $X_{0}=R, X_{k}=\bigotimes_{1 \leqslant i \leqslant k} K_{i}$ образуют цепочку.

ПримеР 10. Все негоренштейновы колшца с $\mathfrak{m}^{2}=0$ являются $S D(1)$-полными. Кольцо с $\mathfrak{m}^{3}=0$ является $S D(2)$-полньм, если существует нетривиальный полудуализирующий $R$-модуль. Кольцо $\bigotimes_{k}^{1 \leqslant i \leqslant n} k \ltimes k^{a_{i}}$ является $S D(n)$-полным в соответствии с [5].

ПредЛОЖЕНИЕ 11. Для $S D(n)$-полного кольца модуль $\bigotimes K_{i}$ является дуализирующuм.

Численные инварианты $S D(n)$-полных колец устроены так же просто, как и для кольца $\bigotimes_{k}^{1 \leqslant i \leqslant n} k \ltimes k^{a_{i}}$, в частности, справедливо следующее предложение.

ПреДЛОЖениЕ 12. Если $R-S D(n)$-полное кольцо, $K_{1}, \ldots K_{n}$ - соответствующие полудуализирующие модули, то для любого $i$ ряд Басса модуля $K_{-i}=\bigotimes_{j \neq i} K_{j}$ есть $I_{K_{-i}}(t)=\frac{\mu^{0}\left(K_{-i}\right)-t}{1-\mu^{0}\left(K_{-i}\right) t}$, а ряд Пуанкаре $K_{i}$ есть $P_{K_{i}}(t)=\frac{\beta_{0}\left(K_{i}\right)-t}{1-\beta_{0}\left(K_{i}\right) t}$.

Для случая $S D(2)$-полных колец можно получить дополнительную информацию - структура таких колец оказывается похожей на структуру колец $\mathrm{cm}^{3}=0$ над которыми имеется нетривильный модуль нулевой $G$-размерности (см. [7]):

ПреДЛОЖЕниЕ 13. Если $R-S D(2)$-полная алгебра над полем, $K$ - нетривиальный полудуализирующий модуль над ней, то на алгебре и на модуле имеется естественная градуировка, причем функции Гильберта имеют вид $\mathrm{H}_{R}(t)=\left(1+\beta_{0}(K) t\right)\left(1+\mu^{0}(K) t\right)$, $\mathrm{H}_{K}(t)=\left(\beta_{0}(K)+t\right)\left(1+\mu^{0}(K) t\right)$.

ПредЛОЖЕнИЕ 14. $S D(2)$ - полная алгебра над полем козюлева, m.e. $\operatorname{Ext}_{R}^{i}(k, k)_{j}=0$ для $i \neq j$.

\section{СПИСОК ЛИТЕРАТУРЫ}

[1] L.W. Christensen // Trans. Amer. Math. Soc. 2001. V. 353. №5. P. 1839-1883. [2] А. А. Герко // Матем. сб. 2001. Т. 192. № 8. С. 79-94. [3] M. Auslander, M. Bridger // Mem. Amer. Math. Soc. 1969. V. 94. [4] L. L. Avramov, H.-B. Foxby // Proc. London Math. Soc. (3). 1997. V. 75. № 2. Р. 241-270. [5] А. А. Герко. Об удобных модулях и $G$-совершенных идеалах // УМH. 2001. T. 56. №4. C. 141-142. [6] T. Araya, R. Takahashi, Y. Yoshino. Homological invariants associated to semidualizing bimodules // Preprint, 2002. [7] Y. Yoshino // NATO Sci. Ser. II Math. Phys. Chem. 2003. V. 115. P. 255-273. 